ELECTRONIC RESEARCH ANNOUNCEMENTS OF THE AMERICAN MATHEMATICAL SOCIETY

Volume 11, Pages 34-39 (May 10, 2005)

S $1079-6762(05) 00144-7$

\title{
COMPLEX GROUP ALGEBRAS OF FINITE GROUPS: BRAUER'S PROBLEM 1
}

\author{
ALEXANDER MORETÓ
}

(Communicated by David J. Benson)

\begin{abstract}
Brauer's Problem 1 asks the following: what are the possible complex group algebras of finite groups? It seems that with the present knowledge of representation theory it is not possible to settle this question. The goal of this paper is to announce a partial solution to this problem. We conjecture that if the complex group algebra of a finite group does not have more than a fixed number $m$ of isomorphic summands, then its dimension is bounded in terms of $m$. We prove that this is true for every finite group if it is true for the symmetric groups.
\end{abstract}

\section{INTRODUCTION}

Let $G$ be a finite group and $\operatorname{Irr}(G)=\left\{\chi_{1}, \ldots, \chi_{k}\right\}$ the set of irreducible characters of $G$. Put $\chi_{i}(1)=n_{i}$. Following B. Huppert, we say that $\left(n_{1}, \ldots, n_{k}\right)$ is the degree pattern of $G$. In recent years much information has been obtained on the possible sets of character degrees of finite groups, especially in the solvable case (even though a complete classification of such sets seems to be very far away). However, as pointed out by Huppert, almost nothing is known about Brauer's Problem 1 (see [2]), which asks the following: What are the possible degree patterns of finite groups? As is well known, if $\left(n_{1}, \ldots, n_{k}\right)$ is the degree pattern of $G$, the complex group algebra of $G$ is $\mathbb{C} G=\bigoplus_{i=1}^{k} \mathrm{M}_{n_{i}}(\mathbb{C})$. So knowing the possible degree patterns of finite groups is equivalent to knowing the possible isomorphism types of complex group algebras.

Even though, in our opinion, with the present knowledge of representation theory it is impossible to settle Brauer's Problem 1, we think that it is possible to obtain significant restrictions on the structure of the complex group algebras. The goal of this note is to provide the first such restriction. For the sake of discussion, we state the following.

Conjecture A. The $\mathbb{C}$-dimension of the complex group algebra of any finite group $G$ is bounded in terms of the maximum number of isomorphic summands in the decomposition $\mathbb{C} G=\bigoplus_{i=1}^{k} \mathrm{M}_{n_{i}}(\mathbb{C})$.

In other words, Conjecture A says that the order of a finite group is bounded in terms of the largest multiplicity of its irreducible character degrees. Our main

Received by the editors October 12, 2004.

1991 Mathematics Subject Classification. Primary 20C15.

Research supported by the Basque Government, the Spanish Ministerio de Ciencia y Tecnología, grant BFM2001-0180, and the FEDER.

(c)2005 American Mathematical Society Reverts to public domain 28 years from publication 
results are the following. As usual, we say that a quantity is $\left(a_{1}, \ldots, a_{l}\right)$-bounded if it is bounded by some real-valued function that depends on $a_{1}, \ldots, a_{l}$.

Theorem B. Conjecture A holds for every finite group if it holds for the symmetric groups.

Theorem C. Let $G$ be a finite group and assume that $G$ does not contain an alternating group bigger than $\operatorname{Alt}(t)$ as a composition factor. If the largest multiplicity of a character degree is $m$, then the order of $G$ is $(m, t)$-bounded.

Unfortunately, we have been unable to prove that Conjecture A holds for the symmetric groups. This seems to be a difficult number-theoretic problem. Note that an immediate consequence of Theorem $\mathrm{C}$ is that Conjecture A holds for solvable groups. (Actually, we prove this result in our way toward a proof of Theorems B and C.)

In the next section we will outline the proof of Theorems B and C. Full details will appear elsewhere. In Section 3, we discuss Conjecture A for symmetric groups.

I thank the referee for a number of useful comments that have improved the readability of this paper.

\section{Proof of Theorems B And C}

The first step in the proof of Theorems B and C is the proof that Conjecture A holds for solvable groups. First, we note that the hypothesis of this conjecture is inherited by quotients. In the key lemma, we show that the number of primes that divide the order of a solvable group that satisfies the hypothesis is bounded. It is an application of results and ideas from [4].

Lemma 2.1. Let $G$ be a solvable group with at most $m$ irreducible characters of each degree and let $p$ be a prime divisor of $|G|$. Then $p$ is m-bounded.

Actually, in this lemma we are just using that for any character $\chi \in \operatorname{Irr}(G)$, the field $\mathbb{Q}(\chi)$ is an extension of $\mathbb{Q}$ of degree $\leq m$. This follows from the fact that all the Galois conjugate characters have the same degree.

By Gaschutz's theorem, if $G$ is a solvable group, then $G / F(G)$ acts faithfully and completely reducibly on $F(G) / \Phi(G)$. This is the context in which we apply the following lemma. We say that a finite module $V$ for a group $G$ has mixed characteristic if $V$ is an abelian group all of whose Sylow subgroups are elementary abelian groups. For such a $G$-module $V, r(G, V)$ stands for the number of orbits in $V$ under the action of $G$. For a solvable group $G$, we write $\operatorname{dl}(G)$ to denote the derived length of $G$.

Lemma 2.2. Let $G$ be solvable and let $V$ be a finite faithful completely reducible $G$-module (possibly in mixed characteristic). Then there exist constants $C_{1}$ and $C_{2}$ such that

$$
\operatorname{dl}(G) \leq C_{1} \log \log r(G, V)+C_{2}
$$

if $r(G, V)>1$, and $\operatorname{dl}(G) \leq C_{2}$ if $r(G, V)=1$.

Proof. This immediate consequence of Theorem 2.4 of [1] appears as Theorem 7.2 in [13].

Finally, we need the following result, which is the nilpotent case of Conjecture A. 
Lemma 2.3. Let $G$ be a nilpotent group and assume that the largest multiplicity of a character degree is $m$. Then $|G|$ is m-bounded.

Proof. This is Corollary 1.12 of [10].

With these ingredients, the idea of the proof of Conjecture A in the solvable case is the following. We consider the solvable groups $G$ with at most $m$ irreducible characters of any given degree. We want to see that $|G|$ is $m$-bounded. First, we look at the solvable groups with fixed derived length $k$. An easy inductive argument shows that for these groups the order of $G$ is $(m, k)$-bounded. Thus, if we want to find a counterexample to Conjecture A for solvable groups, we need to consider groups of arbitrarily large derived length. Indeed, by Clifford's theory and Lemma 2.3, we need to consider groups $G$ such that $\operatorname{dl}(G / F(G))$ is arbitrarily large. Now applying Lemma 2.2 to the action of $G / F(G)$ on $F(G) / \Phi(G)$, we deduce that the number of orbits in this action is doubly exponential in $\operatorname{dl}(G / F(G))$. The inductive argument mentioned above shows that, in fact,

$$
|G: F(G)| \leq m^{4^{\mathrm{dl}(G / F(G))}} .
$$

This together with Lemma 2.1 leads to the fact that the number of divisors of $|G: F(G)|$ is bounded by a function of the form

$$
|G: F(G)| \leq 4^{\mathrm{dl}(G / F(G))} \cdot f(m)
$$

for some function $f$ that only depends on $m$. Since the number of orbits of $G / F(G)$ on $F(G) / \Phi(G)$, and hence on $\operatorname{Irr}(F(G) / \Phi(G)$ ), is doubly exponential in $\operatorname{dl}(G / F(G))$, we deduce that the number of irreducible characters of $G$ whose degree divides $|G: F(G)|$ is doubly exponential in $\operatorname{dl}(G / F(G))$. We conclude that, since $\operatorname{dl}(G / F(G))$ is arbitrarily large, some of the irreducible character degrees occur with multiplicity larger than $m$. This contradiction concludes the proof of Conjecture A for solvable groups.

The next step of the proof of Conjecture A is for the simple groups of Lie type. We will present a proof, due to G. Malle, that gives pretty good explicit bounds. An independent proof has been found in [12]. We will give the proof in a series of lemmas. The idea is to find many Galois conjugate characters.

Lemma 2.4. Let $s \in \operatorname{GL}\left(n, \overline{\mathbb{F}}_{q}\right)$ be semisimple. Then $s$ is conjugate to at most $n$ ! of its powers. If moreover all eigenvalues of $s$ are powers of one among them, then $s$ is conjugate to at most $n$ of its powers.

Lemma 2.5. The group $\operatorname{GL}(n, q)$ contains a semisimple element $s$ of order $q^{n}-1$ all of whose eigenvalues are powers of one among them.

Lemma 2.6. Suppose $G=\mathrm{SL}(n, q), \mathrm{SU}(n, q), \mathrm{Sp}(2 n, q), \mathrm{SO}(2 n+1, q), \mathrm{SO}^{+}(2 n, q)$, $\mathrm{SO}^{-}(2 n, q)$. Then $G$ contains a semisimple element of order $\left(q^{n}-1\right) /(q-1)$, $q^{[n / 2]}-1, q^{n}-1, q^{n}-1, q^{n}-1, q^{n-1}-1$ conjugate to at most $n, n, 2 n, 2 n+1,2 n, 2 n$ of its powers.

Corollary 2.7. Suppose $G=\operatorname{PSL}(n, q), \operatorname{PSU}(n, q), \operatorname{PSp}(2 n, q), \operatorname{PSO}(2 n+1, q)$, $\mathrm{PSO}^{+}(2 n, q), \mathrm{PSO}^{-}(2 n, q)$. Then $G$ contains a semisimple element of order at least $\left(q^{n}-1\right) / n(q-1),\left(q^{[n / 2]}-1\right) / n,\left(q^{n}-1\right) / 2,\left(q^{n}-1\right) / 2,\left(q^{n}-1\right) / 2,\left(q^{n-1}-1\right) / 2$ conjugate to at most $n, n, 2 n, 2 n+1,2 n, 2 n$ of its powers.

These results follow from easy linear algebra arguments. With them and DeligneLusztig theory, we can prove the main result for the classical groups of Lie type. 
Theorem 2.8. Suppose $G=\operatorname{PSL}(n, q), \operatorname{PSU}(n, q), \operatorname{PSp}(2 n, q), \operatorname{PSO}(2 n+1, q)$, $\mathrm{PSO}^{+}(2 n, q), \mathrm{PSO}^{-}(2 n, q)$. Then the largest multiplicity of the character degrees of $G$ is at least $\varphi\left(q^{n}-1\right) / n^{2}(q-1), \varphi\left(q^{[n / 2]}-1\right) / n^{2}, \varphi\left(q^{n}-1\right) / 4 n, \varphi\left(q^{n}-1\right) / 2(2 n+1)$, $\varphi\left(q^{n}-1\right) / 4 n, \varphi\left(q^{n-1}-1\right) / 4 n$.

Theorem 2.9. Conjecture A holds for simple groups of Lie type.

Sketch of proof. For classical groups, this follows easily from Theorem 2.8 using the fact that for any $\varepsilon>0, \varphi(k) / k^{1-\varepsilon} \rightarrow \infty$ as $k \rightarrow \infty$ (see Theorem 327 of [6]).

For exceptional groups, we can use, for example, that $(P) \operatorname{SL}(2, q) \leq G(q)$ for $G(q)$ of exceptional type different from ${ }^{2} B_{2}(q)$ (see the Dynkin diagram) and $G(q) \leq$ $\operatorname{GL}(a, q)$ for some small $a$. For instance, $(P) \mathrm{SL}(2, q) \leq E_{8}(q) \leq \mathrm{GL}(248, q)$. It is easy to see using the first part of Lemma 2.4 that $E_{8}(q)$ contains a semisimple element of order $(q-1) / 2$ conjugate to at most (248)! of its powers. Now, use Deligne-Lusztig theory.

Finally, we consider the groups ${ }^{2} B_{2}(q)$. It is well known that they have $(q-2) / 2$ characters of degree $q^{2}+1$ (see Theorem XI.5.10 of [8]). The result follows.

Now, we can begin to work toward the proof of the general case of Theorems B and C. We need some more lemmas. The first one is a non-trivial number-theoretic result. Given an integer $n$, we write $d(n)$ to denote the number of divisors of $n$.

Lemma 2.10. $\quad$ (i) If $\varepsilon>0$, then $d(n)<2^{(1+\varepsilon) \log n / \log \log n}$ for all $n>n_{0}(\varepsilon)$.

(ii)

$$
\lim _{n \rightarrow \infty} \frac{d(n !)}{2^{\left.\frac{c}{(\log n !} \log n !\right)^{2}}}=1
$$

where $c$ is some constant.

Proof. The first part is Theorem 317 of [6]. The second part is in [3].

We need one more lemma on the characters of the simple groups.

Lemma 2.11. Let $S$ be a finite simple group. Then there exists a non-principal irreducible character of $S$ that extends to $\operatorname{Aut}(S)$.

In the proof of Theorem $\mathrm{C}$, the following result is also necessary.

Theorem 2.12. Let $G$ be a permutation group on a set $\Omega$ of cardinality $k$, and assume that $G$ does not contain any alternating group bigger than $\operatorname{Alt}(t)$ as a composition factor. Then the number of orbits of $G$ on the power set $\mathcal{P}(\Omega)$ is at least $a^{k / t}$ where $a>1$ is some constant.

Proof. This appears in [1].

Now we will sketch the proof of Theorem C.

Sketch of proof of Theorem C. Using the solvable case and Clifford's theory, we may assume that $F(G)=1$. Hence, $G$ is isomorphic to a subgroup of the automorphism group of its socle, so it suffices to bound the cardinality of the socle of $G$.

We have to bound the number of times that a given simple group $S$ appears as a direct factor of the socle and also the order of each of these simple groups. We will just do the first part.

Let $N$ be a normal subgroup of $G$ isomorphic to the direct product of $k$ copies of a non-abelian simple group $S$. We will bound $k$ in terms of $m$. We have that 
$G / C_{G}(N)$ embeds into $\operatorname{Aut}(N) \cong \operatorname{Aut}(S) \imath S_{k}$. Put $H=G / C_{G}(N)$ and view this group as a subgroup of $\operatorname{Aut}(S) \imath S_{k}$. Let $B=H \cap \operatorname{Aut}(S)^{k}$ and note that $H / B$ is a permutation group on $k$ letters. Fix a non-linear character $\varphi \in \operatorname{Irr}(S)$ that extends to $\operatorname{Aut}(S)$ (it exists by Lemma 2.11). Note that the hypotheses of Theorem 2.12 hold. It follows from this result that for some $s$, the number of orbits of $H / B$ on the subsets of cardinality $s$ is at least $a^{k / t} /(k+1)$. Considering the characters of $B$ that extend products of $s$ copies of $\varphi$ and $k-s$ copies of the principal character of $S$, we deduce that there are at least $a^{k / t} /(k+1) H$-orbits of characters of $B$ of the same degree. Using Corollary 11.29 of [9], it follows that $H$ (and hence $G$ ) has at least $a^{k / t} /(k+1) d(k !)$ characters of the same degree. Using part (ii) of Lemma 2.10 and Stirling's formula, one can see that this quotient goes to infinity as $k$ goes to infinity. It follows that $k$ has to be bounded in terms of $m$, as desired.

Once we have proved Theorem C, Theorem B will be an immediate consequence of the following lemma. Given a group $G$, we write $m(G)$ to denote the largest multiplicity of the character degrees of $G$.

Lemma 2.13. Assume that $\operatorname{Alt}(t)$ is a composition factor of a finite group $G$ for some $t>6$. Then

$$
m(G) \geq m(\operatorname{Alt}(t)) / 4 .
$$

In the proof of this lemma, we are using that any invariant character of the base group of a wreath product extends to the whole group.

\section{Symmetric Groups}

The goal of this section is to discuss Conjecture A for symmetric groups. As we will see, this is a combinatorial problem. It is well known that the number of irreducible characters of $S_{n}$ is the number of partitions of $n$. A. Young found a description of the irreducible characters in terms of the partitions of $n$.

Given a partition of $n, \mu=\left(a_{1}, \ldots, a_{t}\right)$, with $a_{1} \geq a_{2} \geq \cdots \geq a_{t}$, the Young diagram associated to $\mu$ is an array of $n$ nodes with $a_{i}$ nodes in the $i$ th row. We assign numbers to the rows and columns and coordinates to the nodes. The hook number $H(i, j)$ of the node $(i, j)$ is the number of nodes to the right and below the node $(i, j)$, including the node $(i, j)$. The degree of the character $\chi_{\mu}$ associated to the partition $\mu$ is given by the hook length formula

$$
\chi_{\mu}(1)=\frac{n !}{\prod_{i, j} H(i, j)} .
$$

This description of the degrees was obtained by J. Frame, G. de B. Robinson and R. Thrall in [5].

Therefore, Conjecture A can be restated as follows. Given an integer $n$, let $m(n)$ be the largest number of partitions of $n$ with the same product of hook numbers.

Conjecture $\mathbf{A}^{\prime} . m(n) \rightarrow \infty$ as $n \rightarrow \infty$.

Computer calculations suggest that this is the case, but it seems to be difficult to find a proof. Just to find a proof of the weaker result $\lim \sup m(n)=\infty$ would be interesting. 


\section{REFERENCES}

[1] L. Babai, L. Pyber, Permutation groups without exponentially many orbits on the power set, J. Combin. Theory Ser. A 66 (1994), 160-168. MR.1273297 (95a:20002)

[2] R. Brauer, Representations of finite groups, Lectures on Modern Mathematics, Vol. I, New York, 1963. MR0178056 (31:2314)

[3] P. Erdős, S. Graham, A. Ivić, C. Pomerance, On the number of divisors of $n$ !, Analytic Number Theory, Progr. Math. 138, Birkhäuser, Boston, 1996. MR.1399347(97d:11142)

[4] E. Farias e Soares, Big primes and character values for solvable groups, J. Algebra 100 (1986), 305-324. MR0840579 (87g:20011)

[5] J. S. Frame, G. de B. Robinson, R. M. Thrall, The hook graphs of the symmetric group, Canadian J. Math. 6 (1954), 316-324. MR0062127|(15:931g)

[6] G. Hardy, E. Wright, An Introduction to the Theory of Numbers, Clarendon Press, Oxford, 1979. MR0568909 (81i:10002)

[7] B. Huppert, Research in representation theory at Mainz (1984-1990), Representation Theory of Finite Groups and Finite-Dimensional Algebras, Progr. Math. 95, Birkhäuser, Boston, 1991. MR:1112156 (92c:20011)

[8] B. Huppert, N. Blackburn, Finite Groups III, Springer-Verlag, New York, 1982. MR0662826 (84i:20001b)

[9] I. M. Isaacs, Character Theory of Finite Groups, Dover, New York, 1994. MR1280461

[10] A. Jaikin-Zapirain, On the number of conjugacy classes of finite $p$-groups, J. London Math. Soc. 68 (2003), 699-711. MR2009445

[11] T. M. Keller, Orbits in finite group actions, Groups St. Andrews 2001 in Oxford. Vol II, pp. 306-331, Cambridge University Press, Cambridge, 2003. MR2051537 (2005b:20023)

[12] M. Liebeck, A. Shalev, Character degrees and random walks in finite groups of Lie type, preprint.

[13] A. Moretó, J. Sangroniz, On the number of conjugacy classes of zeros of characters, Israel J. Math. 142 (2004), 163-187. MR2085714 (2005e:20011)

Departament d’Àlgebra, Universitat de València, 46100 Burjassot, València, SPAin E-mail address: Alexander.Moreto@uv.es 